Sanz-Hernández, A; Alcalá-Martínez, L; Bacallao-Pino, L

Comunicación pública de la ciencia, cultura científica y sentido de localidad. El caso de la ciudad de Teruel, España

Revista Latina de Comunicación Social, núm. 69, enero-diciembre, 2014, pp. 618-636 Laboratorio de Tecnologías de la Información y Nuevos Análisis de Comunicación Social

Canarias, España

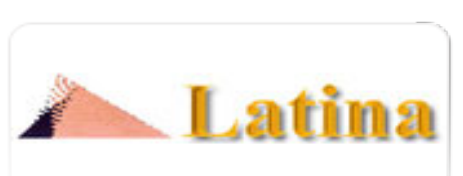

Revista Latina de Comunicación Social,

ISSN (Versión electrónica): 1138-5820

jpablos@ull.es

Laboratorio de Tecnologías de la Información y

Nuevos Análisis de Comunicación Social

España

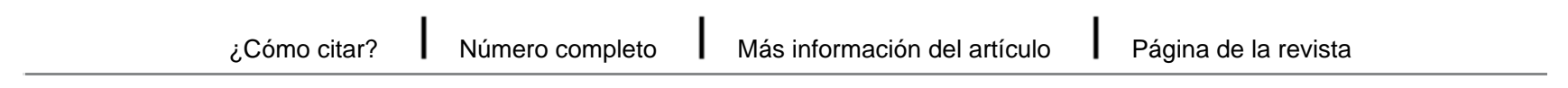

\title{
www.redalyc.org
}

Proyecto académico sin fines de lucro, desarrollado bajo la iniciativa de acceso abierto 


\title{
Cómo citar este artículo / Referencia normalizada
}

A Sanz-Hernández, L Alcalá-Martínez, L Bacallao-Pino (2014): “Comunicación pública de la ciencia, cultura científica y sentido de localidad. El caso de la ciudad de Teruel, España”.

Revista Latina de Comunicación Social, 69, pp. 618 a 636.

http://www.revistalatinacs.org/069/paper/1027_UNAM/30es.html

DOI: $\underline{10.4185 / R L C S-2014-1027}$

\section{Comunicación pública de la ciencia, cultura científica y sentido de localidad. El caso de la ciudad de Teruel, España}

\section{Public communication of science, scientific culture and sense of locality. The case of the city of Teruel, Spain}

\begin{abstract}
A Sanz-Hernández [ $\underline{\mathrm{CV}}$ ] [ $\mathrm{ORCID}$ ] [ $\mathrm{C} \underline{\mathrm{GS}}]$ Profesora del Departamento de Psicología y Sociología - Universidad de Zaragoza, UNIZAR, España - alexsanz@unizar.es
\end{abstract}

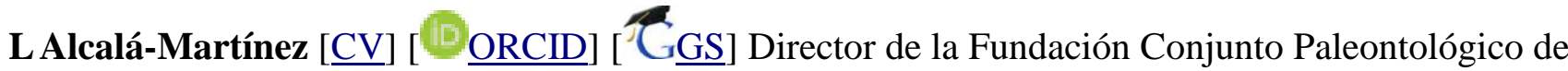
Teruel-Dinópolis, España - alcala@fundaciondinopolis.org

L Bacallao-Pino [CV] [ $\mathrm{CRCID}$ ] [ G GS ] Becario postdoctoral del Centro de Investigaciones sobre América Latina y el Caribe - Universidad Nacional Autónoma de México, UNAM; México lazaro_bacallao@biari.brown.edu

\begin{abstract}
s
[ES] Introducción. Se analiza la mediación del sentido de localidad en las articulaciones entre comunicación pública de la ciencia y percepción social de la ciencia, como parte de los procesos de configuración de la cultura científica. Metodología. Se procede a un estudio de caso de la ciudad de Teruel, en España, mediante una encuesta semiestructurada y entrevistas. Resultados. Las principales consecuencias de la mediación del sentido de localidad en estos procesos se expresa en aspectos como la valoración sobre la comunicación de la ciencia en medios locales, las opiniones sobre las instituciones científicas turolenses y su impacto en el desarrollo local, o el compromiso con aumentar la cultura científica en la ciudad. Discusión. Visto lo anterior, se examinan las principales dimensiones (comunicativa, emocional, en las interrelaciones individuo/colectivo) en las que se ejerce esa mediación. Conclusiones. Al final encontramos que la dimensión local ejerce una mediación compleja en la cultura científica.
\end{abstract}

[EN] Introduction. This article analyses how the sense of localness mediates the articulations of the public communication and the social perception of science, as part of the configuration of scientific 
culture. Method. The analysis is based on the case study of the Spanish city of Teruel, which is examined by means of a semi-structured questionnaire and interviews. Results. The main consequences of the mediation exercised by the sense of localness in the public communication and the social perception of science occurred in such areas as the assessment of the communication of science in local media, the opinions on Teruel's scientific institutions and their impact on local development, and people's commitment to increase scientific culture in the city. Discussion. Taking into consideration the previous results, the article examines the main dimensions (communicative, emotional, individual/collective relationships) in which the local dimension mediates those articulations. Conclusions. The local dimension mediates the configuration of scientific culture in a complex way.

\section{Keywords}

[ES] Comunicación pública de la ciencia; cultura científica; percepción social de la ciencia; localidad.

[EN] Public communication of science; scientific culture; social perception of science; localness.

\section{Contents}

[ES] 1. Introducción. 2. Método. 2.1. Estrategias metodológicas. 2.2. Población y muestra. 2.3. Instrumentos de recogida de información. 2.4. Procedimiento. 3. Resultados. 4. Discusión y conclusiones. 5. Notas. 6. Referencias bibliográficas.

[EN] 1. Introduction. 2. Method. 2.1. Methodological strategies. 2.2. Population and sample. 2.3. Data collection tools. 2.4. Procedure. 3. Results. 4. Discussion and conclusions. 5. Notes. 6. List of references.

Traducción de Lázaro M. Bacallao-Pino, Ph.D. (National Autonomous University of Mexico).

\section{Introducción}

La ciencia y la tecnología han tenido un papel social exponencialmente creciente en la modernidad, hasta llegar a las sociedades contemporáneas, en las cuales se asiste a la emergencia de campos científicos como la biotecnología o la genómica, o el extenso impacto social de ciertas áreas tecnológicas como las tecnologías de la información y la comunicación (TIC). La presencia de la actividad científica y tecnológica en las sociedades actuales se extiende a la casi totalidad de las esferas de la vida cotidiana: la educación, la salud, la alimentación, el transporte, la vivienda, el ocio, el trabajo, la economía y el trabajo. Sin embargo, en contraposición a ello, ciertas investigaciones han señalado el desinterés, incluso entre los jóvenes, por los temas científicos (Gil et al., 2005), llegándose a considerar imposible la alfabetización científica del conjunto de la ciudadanía (Gil y Vilches, 2004).

Frente a tal escenario, se plantea la necesidad de aumentar la cultura científica ciudadana -por medio de acciones como la divulgación a través de los medios de comunicación, denominada comunicación pública de la ciencia-, al considerarse las importantes contribuciones de la ciencia a la cultura ciudadana, entre las que se mencionan la participación en los procesos de toma de decisiones en los temas relacionados con el desarrollo tecnocientífico, así como su contribución a la formación de un espíritu crítico en las ciudadanas y ciudadanos (Gil y Vilches, 2006). Las interrelaciones entre ciencia y sociedad no resultan lineales y simples pues, como afirma Magro Mazo (2008), "ya no basta la afirmación de que más conocimiento, generará riqueza y por tanto bienestar social. Las 


\section{Revista Latina de Comunicación Social \# 069 - Páginas 618 a 636 \\ Investigación financiada | DOI: 10.4185/RLCS-2014-1027 | ISSN 1138-5820 | Año 2014}

relaciones entre ciencia y sociedad se muestran como algo mucho más complejo que esta sencilla ecuación".

La cultura científica ha sido definida por Vaccarezza (2008: 110), “como comprensión de la dinámica social de la ciencia, de manera que se tejen, en una interrelación entre productores de conocimientos científicos y otros grupos sociales, todos ellos como partícipes del devenir de la cultura, produciendo significados cuyos orígenes y justificaciones provienen desde distintas prácticas, intereses, códigos normativos y relaciones de poder, entendiéndose como un devenir continuo". La dimensión comunicativa resulta central en esta conceptualización, en tanto se trata de un concepto intrínsecamente relacional, asociado a un proceso de producción de sentido en torno a la actividad científica.

Esta relación entre comunicación y ciencia ha estado marcada tradicionalmente por una tendencia predominante ligada a cierta concepción pedagógica y la divulgación, que reduce los actores de la comunicación a tres -los investigadores científicos y sus instituciones, el público y los pedagogos o periodistas, mediadores con el encargo de interpretar y dar forma a los mensajes científicos para garantizar su comprensión por parte de los primeros-, estableciendo una relación unidireccional entre estos, según el esquema Emisor $\rightarrow$ Mensaje $\rightarrow$ Receptor. En correspondencia con este enfoque, ha existido en los investigadores científicos el criterio de considerar la divulgación como "una misión imposible o, aún más, como una actividad bastarda, sospechosa de trasmitir cualquier cosa menos ciencia" (Fayard, 2004: 14).

Frente a esa comprensión tradicional de la relación entre ciencia y comunicación, este autor señala la necesidad de un tránsito desde un paradigma divulgativo a otro de mediatización, a partir de un cambio en el enfoque en la relación entre los actores del proceso y de estos con el propio conocimiento y la cultura. La transición desde una perspectiva de divulgación a otra de comunicación pública para la ciencia, supone superar su reducción a una simple transferencia de conocimiento, que de hecho con frecuencia produce efectos contrarios al propósito inicial de acercar, compartir y estimular.

Este cambio en el paradigma de comprensión de la comunicación de la ciencia, se corresponde con el incremento en los niveles de importancia de la percepción pública de la ciencia, proceso que viene de la mano, a su vez, del aumento del impacto de la ciencia y la tecnología en las más variadas dimensiones sociales. Ese interés aparece vinculado, además, a tres cuestiones interrelacionadas: el interés de la comunidad científica por mantener niveles de financiamiento público necesarios, el surgimiento de movimientos y organizaciones sociales críticos al desarrollo tecnocientífico y el diseño de políticas públicas dirigidas a actuar sobre la comprensión social de la ciencia y la sensibilización ciudadana al respecto (Polino et al., 2003). Expresión de este interés ha sido la realización de estudios sobre percepción pública y cultura científica, una práctica que se ha extendido entre los organismos de ciencia y tecnología de diferentes países desde mediados de los años 70 .

El surgimiento de los estudios sobre percepción pública y cultura científica tuvo como principal objetivo devolverle a la ciencia la confianza de la sociedad, afectada por el desarrollo de tecnologías como la nuclear o los agrotóxicos, así como fenómenos como la crisis energética o el cambio climático (Polino et al., 2003). Sin embargo, como precisan estos autores, tales estudios han transitado por tres etapas, con objetivos de investigación que responden a necesidades diferentes. A ese propósito inicial de recuperar la confianza social, presente hasta mediados de los años 80, se añadió desde esta fecha y hasta finales de esa década un segundo objetivo: reinstalar el debate acerca de la cantidad y calidad de la alfabetización científica de la población. 


\section{Revista Latina de Comunicación Social \# 069 - Páginas 618 a 636 \\ Investigación financiada | DOI: 10.4185/RLCS-2014-1027 | ISSN 1138-5820 | Año 2014}

A partir de los años 90, en una tendencia creciente, este tipo de estudios asumió una nueva proyección, de carácter estratégico, como parte del propósito de incrementar la participación social vinculada a la actividad científica y tecnológica, democratizándola sobre la base de un aumento del control social de la ciencia y la tecnología, que pasaría -entre otras cuestiones- por una mejor información a los ciudadanos y la articulación de causes institucionales para la expresión de aquellos en relación con estos temas (García Galindo y Moreno Castro, 1999).

Al respecto, los indicadores de percepción social de la ciencia tienen como meta, actualmente, "evaluar y observar las relaciones entre tres dimensiones: la percepción pública, la cultura científica y la participación ciudadana, o lo que es lo mismo, medir la sensibilización, los conocimientos y la participación" (Magro Mazo, 2008: 146). Sin embargo, como plantea este autor, las críticas a los instrumentos aplicados en los estudios señalan la continuidad de su anclaje en un paradigma de la actividad científica ya superado, que privilegia la componente comunicativa en la indagación, en detrimento de las dimensiones participativa y democrática.

En tal sentido, el propósito de este artículo es examinar la mediación que ejerce la dimensión local escenario privilegiado de la participación ciudadana (Font et al., 2002)- en las interrelaciones entre comunicación pública de la ciencia y percepción social de esta que se dan en las dinámicas de configuración de la cultura científica. Si bien la complejidad y dependencia del contexto en la comunicación pública de la tecnociencia ha sido subrayado en análisis anteriores (Alcíbar, 2009), no se han encontrado estudios anteriores que examinen específicamente la mediación de lo local.

En consecuencia, su inclusión como dimensión mediadora en el análisis del tema, resulta una perspectiva analítica novedosa, no solo por la relevancia de la participación ciudadana en este nivel, sino también por la particular importancia que adquiere lo local en el mundo contemporáneo como correlato de los procesos globalizadores (Hylland Erikse, 2014), incluyendo la cuestión del ámbito informativo y comunicativo local (Cantalapiedra, 1997).

Tomando este enfoque como fundamento, se propone mostrar que, en determinados contextos en los cuales el sentido de localidad es particularmente relevante para los sujetos, la dimensión local expresada en aspectos como sentimientos de pertenencia, indicadores identitarios, etc.- media de manera significativa los procesos de apropiación de la ciencia y la tecnología, en particular la articulación entre comunicación pública y percepción social de la misma.

Los sentidos de proximidad y de pertenencia asociados a lo local (Pellegrino, 2011) devendrían facilitadores de la emergencia de una visión de la ciencia y la tecnología que trascienda cierta representación social de la ciencia como algo propio de expertos, distante de los individuos, en lugar de como una cuestión cercana, práctica y concreta, que la asume más como producto que en tanto que un proceso desmitificado y abierto a las personas ordinarias (Domínguez-Gutiérrez, 2006).

\section{Método}

En correspondencia con la naturaleza del problema a investigar y los objetivos planteados, se ha seguido una perspectiva metodológica mixta, en la cual se han complementado los enfoques cuantitativo y cualitativo.

Esta articulación, cuyas ventajas han sido destacadas por distintos autores (Creswell y Plano Clark, 2011) en respuesta a la oposición dicotómica entre ambos enfoques, permite una comprensión de los problemas de investigación en la cual se articulan la utilización de estadísticas y la medición de 


\section{Revista Latina de Comunicación Social \# 069 - Páginas 618 a 636 \\ Investigación financiada | DOI: 10.4185/RLCS-2014-1027 | ISSN 1138-5820 | Año 2014}

determinados fenómenos, propios de lo cuantitativo, con la exploración en profundidad de las problemáticas examinadas, y la extracción y comprensión de los significados vinculados a las mismas, característico de lo cualitativo.

\subsection{Estrategia metodológica}

La estrategia metodológica seguida fue el estudio único de caso, cuya selección estuvo determinada por la participación de Teruel en el proyecto europeo Platform of Local Authorities and Communicators Engaged in Science (PLACES), que se enmarca en el $7^{\circ}$ Programa Marco de Investigación de la Unión Europea. Sin embargo, el contexto turolense también resulta relevante para el análisis del tema propuesto.

Teruel es la capital de provincia menos poblada de España (INE, 2009), por lo que estamos ante un entorno urbano singular para el análisis de lo local y al mismo tiempo, dada su condición de capital provincial, con ciertas condiciones de posibilidad para un mayor nivel de impacto de la actividad científica.

Al mismo tiempo, la ciudad cuenta con importantes espacios de carácter científico, como el parque cultural, científico y de ocio Territorio Dinópolis, en el cual se integra la Fundación Conjunto Paleontológico de Teruel. Este espacio ha recibido más de 2 millones de visitantes desde su inauguración en 2001 y, en 2013, recibió cerca de 175 mil visitantes [1], es decir, casi cinco veces la población de la ciudad. La combinación de un entorno urbano singularmente local, una población significativamente envejecida y la presencia de instituciones de carácter científico, hacen de Teruel un caso relevante para el análisis propuesto.

Dada la novedad del tema, se ha planteado realizar un estudio descriptivo, que permita ofrecer una caracterización de cómo media esa dimensión local propia del caso seleccionado en los procesos de configuración de la cultura científica de los habitantes de la ciudad, en particular la articulación entre comunicación pública y percepción social de la actividad científica y tecnológica.

\subsection{Población y muestra}

Con una población de 35961 habitantes, el 48,47\% de los turolenses son hombres y el 51,72\%, mujeres; por edades, el 26,06\% tiene entre 0 y 24 años, el 56,23\% entre 25 y 64 años, y el 17,71 tiene 65 o más (INE, 2013). Para analizar la cultura científica y la percepción social de la ciencia en los habitantes de la ciudad, se ha realizado una encuesta a un total de 656 sujetos mayores de 16 años residentes en Teruel y se han realizado entrevistas en profundidad a personas directamente vinculadas a la actividad científica y tecnológica en el territorio.

La selección de las personas encuestadas no se ha realizado siguiendo criterios de representatividad estadística sino tomando como criterio su pertenencia a las diferentes asociaciones a través de las cuales los habitantes de la ciudad participan. De esta forma, se ha identificado un conjunto de espacios de participación ciudadana en la ciudad, de entre cuyos miembros se han seleccionado las personas a encuestar: fundaciones, asociaciones de vecinos, instituciones educativas (institutos y universidad), organizaciones no gubernamentales, asociaciones de empresarios, medios de comunicación y sindicatos. 
Al tomar la pertenencia al tejido asociativo de la ciudad como criterio teórico para la selección muestral se logra, por una parte, superar determinadas críticas a la encuesta como técnica de investigación -en particular, su atomismo y, por consiguiente, el no tener en cuenta las relaciones, interacciones ni grupos sociales (Alvira Martín, 2011:12-13)- y, por otra, se inscribe el estudio en la tendencia - presente desde la década de los 90 en los estudios sobre percepción social de la cienciade contribuir a la participación social y a la democratización de la ciencia y la tecnología, como parte de un proceso de apropiación de la misma. Del igual modo, este criterio es coherente con una propuesta de cuestionario que intenta adecuarse a la complejidad de la mediación de la dimensión local que se propone analizar.

\subsection{Instrumentos de recogida de información}

Para la elaboración del cuestionario semiestructurado aplicado en la encuesta, se han tomado como referencia los cuestionarios aplicados en los estudios de percepción social de la ciencia (López Cerezo et al., 2009), pero el tipo de preguntas planteadas se ha adecuado tanto en su forma como en su contenido al enfoque complejo e integral propuesto.

De esta forma, se han incluido tres tipos de interrogantes específicas, para: 1) conocer las valoraciones de los encuestados acerca de los niveles de cultura científica de la ciudad y del papel de la ciencia en Teruel; 2) recabar información sobre la comunicación pública y la percepción social de la ciencia en los sujetos; y 3 ) indagar en torno a la dimensión local en los sujetos, particularmente en relación con la actividad científica (identificación con la actividad científica local, opiniones acerca de los objetivos, líneas estratégicas, actuaciones y resultados que, a juicio de los sujetos, debería tener la actividad científica y tecnológica para la ciudad, etc.).

En consecuencia, se han incluido preguntas cerradas y abiertas, en un cuestionario semiestructurado que incluye indicadores tanto de comunicación pública y percepción social de la ciencia, como otros relacionados con la dimensión local.

En el caso de las entrevistas, éstas se han realizado a partir de una guía temática abierta, que también ha incluido cuestiones relacionadas con las tres dimensiones incluidas en el cuestionario de la encuesta.

\subsection{Procedimiento}

La elaboración de los instrumentos de recogida de información se realizó a partir de la investigación bibliográfica y/o documental de estudios previos sobre percepción social y comunicación pública de la ciencia. De forma paralela, se identificaron, por una parte, los principales espacios que configuran el tejido asociativo, canalizando la participación en la ciudad de Teruel y, por otra, aquellos sujetos de relevancia por su vinculación con la actividad científica y tecnológica turolense.

Una vez elaborados los instrumentos e identificados los espacios y sujetos, se procedió a aplicar la encuesta y la entrevista. En el caso de la primera, se utilizaron dos canales para su aplicación: cuestionarios impresos y un formulario creado a través de Google Drive. Las entrevistas, por su parte, se realizaron de manera presencial. Una vez obtenidos los datos, se procedió a su análisis.

En el caso de las preguntas estructuradas incluidas en la encuesta, su procesamiento se realizó 


\section{Revista Latina de Comunicación Social \# 069 - Páginas 618 a 636 \\ Investigación financiada | DOI: 10.4185/RLCS-2014-1027 | ISSN 1138-5820 | Año 2014}

mediante Excel, mientras los datos obtenidos en la preguntas abiertas y entrevistas (textos) fueron sometidos a un análisis cualitativo de contenido, que permite identificar significados y sentidos a través de un conjunto de procedimientos interpretativos (Piñuel Raigada, 2002).

Los resultados de ambos procesos fueron triangulados, técnica basada en "la contrastación de la descripción, explicación, y evaluación de los contenidos analizados en una investigación, con otras descripciones, explicaciones y evaluaciones de otras investigaciones independientes realizadas sobre el mismo objeto, o bien, dentro de una misma investigación sobre idéntico objeto, de una combinación de técnicas, entre ellas el análisis de contenido, como medio de dar validez externa a los datos" (Piñuel Raigada, 2002: 14).

\section{Resultados}

Los sujetos encuestados muestran un elevado interés informativo acerca de los temas de ciencia y tecnología. La mayoría de los encuestados $(67,38 \%)$ declara que se informa con una frecuencia elevada sobre temas relacionados con la ciencia y la tecnología: el 32,62\% los hace frecuentemente y el 34,76\% de vez en cuando. Mientras que, por el contrario, apenas el 13,72\% muestra un interés bajo en informarse por estas temáticas $(2,13 \%$, nunca y $11,59 \%$, casi nunca). En un término medio, que se informan al respecto solo a veces, se encuentran casi uno de cada cinco encuestados $(18,75 \%)$. [2].

Por un lado, esta tendencia contradice una posible lectura que asumiría que en Teruel, al formar parte de Aragón, habría un bajo interés por las noticias e informaciones de carácter científico y tecnológico, pues esta Comunidad Autónoma se ubica entre las de más bajo porcentaje en este aspecto en la IV Encuesta de Percepción Social de la Ciencia (FECYT, 2012).

Sin embargo, este comportamiento estaría en relación con la estructura de formación y ocupación de los sujetos encuestados, entre los cuales la mayoría tiene formación universitaria $(51,52 \%)$-seguido, aunque con una notable diferencia, por el bachillerato $(26,52 \%)$ y la formación profesional $(8,99 \%)$-, y ubicados además de manera levemente mayoritaria en el perfil de trabajador asalariado $(50,91 \%)$, seguido -también con una notable diferencia- por el colectivo estudiantil $(28,63 \%)$.

En el sistema comunicativo local turolense, la respuesta a esta necesidad informativa se concentra, sobre todo, en el Diario de Teruel, que publicó desde 2003 hasta 2014 un suplemento semanal, bajo el título Fundamentos paleontológicos, coordinada por la dirección gerencia de la Fundación Dinópolis. Esta singular iniciativa se articuló a partir de las colaboraciones de los científicos de la Fundación hasta alcanzar los 500 números y ha incorporado además, una sección internacional para la divulgación de las riquezas geológicas y paleontológicas de geoparques de toda Europa, en otra iniciativa inédita en este campo. De igual forma, desde hace dos años, y coordinado por el campus de la Universidad de Zaragoza, se publican semanalmente artículos de divulgación científica en el periódico.

En la radio cabe destacar un espacio mensual denominado "Lógica paleontológica" (Radio 5 de Radio Nacional de España en Teruel) mientras en la televisión regional no existen espacios de esta naturaleza, sino solo coberturas puntuales de las actividades vinculadas a la ciencia y la técnica y, de hecho, la televisión aragonesa no respondió positivamente a la creación de una sección vinculada al proyecto PLACES, por considerar que existía poca audiencia potencialmente interesada en el tema. Este panorama mediático local se corresponde, sobre todo en el caso de estos últimos medios, con una tendencia identificada también en los principales diarios de pago generalistas españoles, que 
según estudios anteriores muestran una baja presencia de información científica y carecen de criterios para clasificarla en una sección concreta (Aladro Vico et al., 2014).

En correspondencia con el elevado interés mostrado por los encuestados y esta presencia del tema en el sistema comunicativo local, un alto porcentaje de las personas $(68,60 \%)$ considera que es poco el espacio que los medios de comunicación de Teruel dedican a noticias sobre ciencia y tecnología, mientras que -en el extremo opuesto- apenas el $0,76 \%$ considera que es demasiado el espacio. Se muestra así una necesidad informativa al respecto, que no es satisfecha por parte de los medios locales turolenses.

En tal sentido, resulta pertinente considerar los medios de comunicación a través de los cuales los sujetos declaran que les resultaría más agradable recibir información sobre ciencia y tecnología. Al respecto, todos los medios mencionados (periódico, radio, televisión, Internet y redes sociales, publicaciones especializadas), tienen un nivel de preferencia que supera la media de la escala propuesta (entre 1 y 5), ubicándose siempre por encima del valor correspondiente al "ni me desagradaría ni me agradaría". En todos los casos, exceptuando la radio -cuya moda se ubica en el nivel de "me agradaría" y que, justamente, tiene la media más baja (3,63 puntos)-, la moda corresponde al nivel de "me agradaría mucho".

De manera significativa, los dos medios que mayor media de preferencia presentan son Internet y las redes sociales y la televisión, confirmando la tendencia general, entre los españoles, a informarse sobre temas científica en primer lugar a través de Internet, y en segundo lugar por medio de la televisión, además de la hegemonía de las redes sociales como canal de información científica entre los menores de 25 años (FECYT, 2012). La televisión, en particular, se considera un medio fundamental para la alfabetización científica de la población (Martínez Ruiz et al., 2005), al punto de configurarse en este caso, dadas las especificidades del medio, un forma distinta de comunicar: la divulgación científica audiovisual (Salcedo, 2009).

Esta tendencia en la valoración de los diferentes medios estaría asociada, por una parte, a la composición etárea de la muestra - con un 44,67\% de los encuestados ubicados en la franja de edad joven, entre16 y 35 años- así como a la importante presencia de graduados universitarios y de nivel medio superior y, por otra, al cambio de paradigma en la comunicación pública de la ciencia, desde un enfoque divulgativo unidireccional a otro de mediatización y bidireccional (Fayard, 2004), facilitada en el caso de Internet por la multimedialidad y la interactividad propias de esta.

Gráfico 1. Moda y media de evaluación de los diferentes medios de comunicación, a partir de cuánto gustaría a los encuentados informarse sobre temas científicos a través de cada uno de ellos. Elaboración propia. Leyenda: $1=$ "no me agrada nada"; $2=$ "no me agrada"; $3=$ "ni me agrada ni me desagrada"; $4=$ "me agrada" y $5=$ "me agrada mucho". 


\section{Revista Latina de Comunicación Social \# 069 - Páginas 618 a 636 Investigación financiada | DOI: 10.4185/RLCS-2014-1027 | ISSN 1138-5820 | Año 2014}

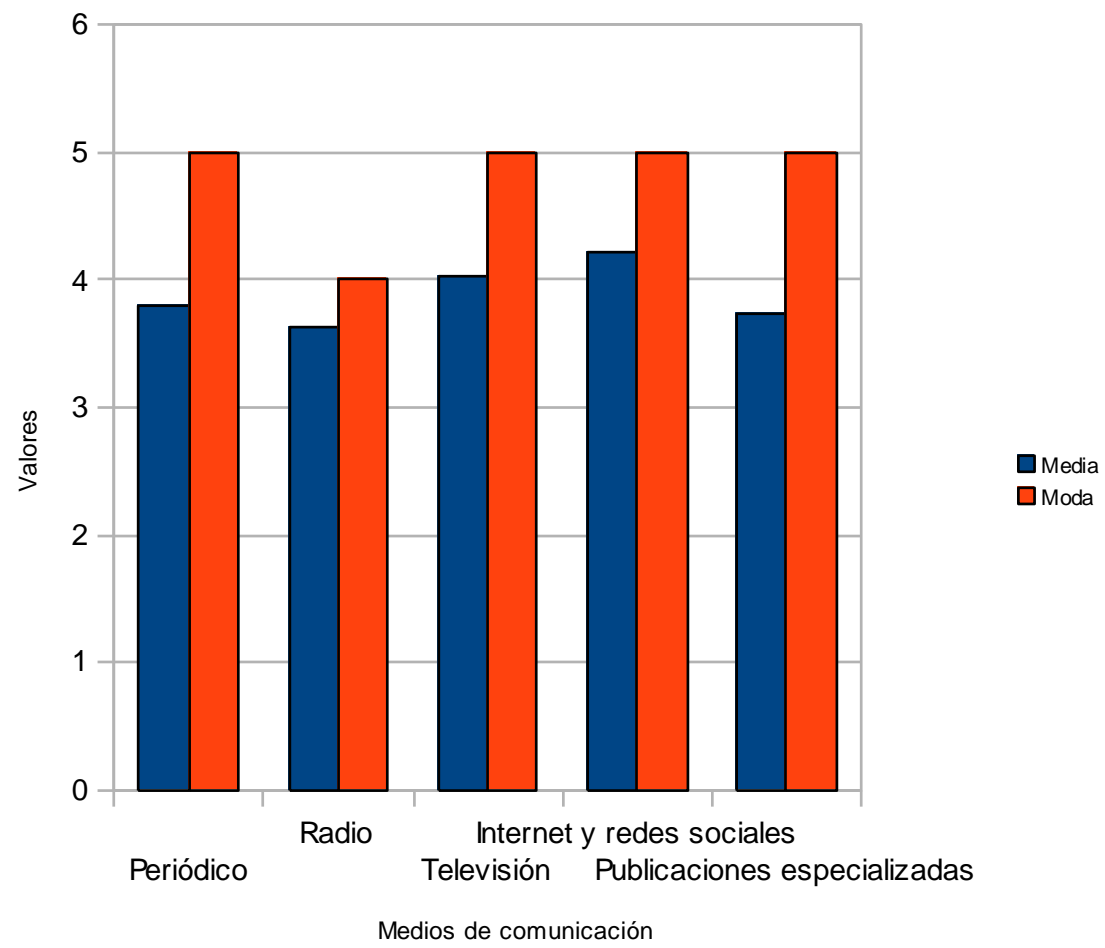

Esa tendencia a preferir una comunicación pública de la ciencia de carácter bidireccional, se confirma con la sugerencia, por parte de los entrevistados, de la creación de espacios comunicativos cara a cara y de carácter presenciales, como charlas, conferencias y cursos, exposiciones, jornadas de divulgación y puertas abiertas, así como acciones locales específicas (publicaciones gratuitas y publicidad en las calles de la ciudad, en los comercios, en las instituciones educativas y científicas).

En tal sentido, además de esa preferencia por una comunicación bidireccional, estas recomendaciones también muestran la preferencia por los espacios comunicativos comunitarios y locales.

Sin embargo, este interés informativo general en temas de ciencia y tecnología, no se corresponde con los niveles de interrelación presencial con las instituciones locales vinculadas a esta actividad. La mayoría de los encuestados declaran que visitan estas instituciones con poca frecuencia: el $23,02 \%$ lo hace a veces, el 34,76\% casi nunca y el 12,80\% nunca. Frente a ello, solo el $8,54 \%$ las visita frecuentemente y el 19,82\% lo hace de vez en cuando [3].

Es decir, más de la mitad de los encuestados $(70,58 \%)$ visitan estas instituciones con una frecuencia baja mientras solo uno de cada $3(28,36 \%)$ lo hace con regularidad, confirmando así, en el caso analizado, una de las paradojas señaladas por Fayard (2004: 17): la falta de correspondencia entre el reconocimiento de la importancia de las ciencias y de las tecnologías por las personas y los bajos niveles de asistencia a los centros de cultura científica.

Esta baja frecuencia de visitas a instituciones que los encuestados asocian a la actividad científica tiene, entre sus explicaciones fundamentales, la percepción que tienen los sujetos de cuáles son las entidades locales vinculadas a la ciencia y la tecnología, así como la orientación externa -hacia el turismo- de las actividades que realizan las mismas, con lo cual se considera que, por ejemplo, en el caso de los museos, es suficiente una sola visita, al no actualizarse estos de manera periódica. Lo local media, de este modo, tanto la representación de lo que es o no científico como la valoración de las actividades que estas realizan. 


\section{Revista Latina de Comunicación Social \# 069 - Páginas 618 a 636 \\ Investigación financiada | DOI: 10.4185/RLCS-2014-1027 | ISSN 1138-5820 | Año 2014}

En contraposición, quienes visitan con más frecuencia estas instituciones -por lo general personas con formación universitaria que realizan actividades vinculadas directa o indirectamente a la investigación-, lo hacen principalmente por motivos personales de carácter profesional o laboral, por lo que en este caso, no es necesario una labor de difusión por parte de aquellas. En este caso, es la dimensión personal -más que la local-la que incide en la frecuencia con la cual se visita este tipo de entidades.

En relación con estas tendencias, los entrevistados subrayan, por un lado, la necesidad de una adecuada estrategia de comunicación con la comunidad de estas entidades y, por otro, la permanencia en muchas personas de una percepción de imposibilidad de acceso o visita a las instituciones vinculadas a la actividad científica o tecnológica.

Estamos en presencia de una cercanía física pero un distanciamiento simbólico de los sujetos con respecto a las organizaciones locales que asocian a estas actividades, que muestra la tendencia a una representación social de la ciencia como cuestión alejada de la vida cotidiana y área exclusiva de expertos (Domínguez-Gutiérrez, 2006).

Efectivamente, solo dos de los tipos de instituciones mencionadas en el cuestionario fueron identificadas con la actividad científica por más de la mitad de los encuestados: los centros de investigación -cuyo nombre mismo lo explicita- y los centros educativos (escuelas, institutos, universidad) y los institutos de estudios, parte de la comunidad educativa, cuyo papel en el desarrollo del conocimiento científico se considera relevante (Martínez Ruiz et al., 2005).

Sin embargo, aquellas instituciones más vinculadas a la actuación cotidiana y local de los individuos, como las empresas y asociaciones, fueron señaladas solo en el $13 \%$ y el $29 \%$ de las respuestas, respectivamente. La cotidianidad local de los sujetos, por consiguiente, no se asocia al quehacer científico y tecnológico, pues no se vinculan a este aquellas instituciones que mayor presencia tienen en el tejido territorial, como pueden ser las empresas o asociaciones. En la medida en que un mayor número de instituciones sean asociadas a esta actividad, más posibilidades habrá de que los sujetos consideran que las visitan con frecuencia.

En el mapa local de la ciencia que dibujan los encuestados, se destacan la Fundación Conjunto Paleontológico de Teruel-Dinópolis, el Centro de Estudios de Física del Cosmos de Aragón (CEFCA), el Campus Universitario local de la Universidad de Zaragoza, el Instituto de Estudios Turolenses, el Museo de Teruel y los institutos de enseñanza secundaria. Solo dos empresas se incluyen -el Grupo Térvalis, dedicado a la producción de fertilizantes, y la Unión Vidriera Aragonesa-, además de la Cámara de Comercio y el Consejo Regulador D.O. Jamón de Teruel.

Tradicionalmente, los estudios sobre percepción social de la ciencia, solicitan a los sujetos valoraciones generales y universalistas sobre la ciencia y la tecnología (López Cerezo et al., 2009). Por el contrario, en este caso se han solicitado evaluaciones sobre la actividad científica en la localidad donde se vive. En tal sentido, el mayor porcentaje de respuestas $(43,29 \%)$ consideró a Teruel como una ciudad atrasada en temas de investigación científica, mientras que, en el extremo opuesto, la respuesta menos frecuente $(6,10 \%)$ la calificó como adelantada en este aspecto. Un $35,98 \%$ de los encuestados opta por una valoración intermedia mientras que el 14,18\% no tiene una opinión formada al respecto. [4]

Los entrevistados consideran que esa tendencia podría tener su explicación en una aplicación o extensión, al ámbito científico-tecnológico, de una imagen generalizada de Teruel como "ciudad atrasada en todo", que por ejemplo, ha tenido que movilizarse socialmente en reclamo de 


\section{Revista Latina de Comunicación Social \# 069 - Páginas 618 a 636 \\ Investigación financiada | DOI: 10.4185/RLCS-2014-1027 | ISSN 1138-5820 | Año 2014}

infraestructuras e inversiones, como en el caso de la Coordinadora Ciudadana Teruel Existe. [5]

En consecuencia, también en este aspecto lo estará, sobre todo tratándose de una actividad inherentemente asociada al desarrollo. Los encuestados, por su parte, fundamentan esta valoración negativa sobre la ciencia y la tecnología turolenses en la desconexión entre ciencia, empresas y administración local, así como en la escasa cultura científica de la población, que obstaculizaría el surgimiento de más iniciativas científicas "desde abajo" (particulares, empresas, asociaciones).

Un aspecto importante en la percepción social de la ciencia que muestran los sujetos, está relacionada con la evaluación que hacen estos de las instituciones científicas turolenses. Al respecto, al comparar la evaluación que hacen las personas encuestadas de sus aportaciones específicas al desarrollo local y la utilidad general para la sociedad de los conocimientos que generan, aunque en ambos casos se valoran positivamente, hay una tendencia a considerar mayor la segunda mientras se resulta más críticos con la evaluación de su aportación local.

En el primer caso, la mayoría de los sujetos declaran no estar "ni de acuerdo ni en desacuerdo" con considerar significativas sus aportaciones, mientras que, con respecto al segundo, la tendencia mayoritaria es a estar "de acuerdo" con que los conocimientos que generan son útiles para la sociedad en general. Esta tendencia se confirma con el hecho de que la inmensa mayoría de los encuestados $(87,04 \%)$ que considera que, en un futuro, el aporte de la ciencia al desarrollo de la ciudad debería ser mayor a como ha sido hasta ahora, mientras que solo un $2,13 \%$ considera que debería ser igual, apenas un $0,76 \%$ opina que debería ser menor y un $8,54 \%$ no tiene una opinión conformada al respecto. [6]

Así mismo, las demandas de los ciudadanos en relación con esas aportaciones futuras de las entidades científicas turolenses al desarrollo local, responden a esa importancia concedida a la dimensión local y, en consecuencia, se vinculan a las problemáticas locales más relevantes en la actualidad.

A juicio de los sujetos, la contribución más importante de la actividad científica estaría vinculada, en primer lugar, a la creación de puestos de trabajo (74,24\% de los encuestados). Se explica que, en un contexto de profunda crisis económica y elevadas tasas de desempleo, la creación de empleo sea la contribución de la actividad científica a la localidad destacada por la mayor cantidad de sujetos. Teruel terminó 2013 con una tasa de paro del 19,83\% y, en marzo de 2014, se registraron 10659 parados en la provincia, siendo la segunda con mayor tasa de paro en la Comunidad aragonesa. [7]

La segunda demanda más relevante, según los resultados de la encuesta aplicada, tiene un carácter general: el 54,27\% de los encuestados considera una importante contribución la realización de innovaciones que sean importantes para la sociedad. El resto de las contribuciones que los encuestados señalan como importantes, reiteran la importancia de lo local, tanto en su dimensión económico-laboral como sociocultural.

Así, el 49,85\% señala como aspecto aportación relevante el aumento de la cultura de los habitantes de la ciudad y el $41,46 \%$ de los encuestados considera prioritario un propósito directamente vinculado al impacto económico de la ciencia, esto es, alcanzar una mayor aplicación de los resultados científico-tecnológicos a las empresas locales.

Otra dimensión relevante, en relación con lo local, es el sentido de pertenencia y la identificación de los sujetos con una comunidad determinada (Morley y Robins, 2013). En consecuencia, se ha indagado en estas cuestiones, en relación con la actividad científica en Teruel. En general, los encuestados muestran un elevado nivel de identificación con el propósito de hacer de Teruel una 


\section{Revista Latina de Comunicación Social \# 069 - Páginas 618 a 636 \\ Investigación financiada | DOI: 10.4185/RLCS-2014-1027 | ISSN 1138-5820 | Año 2014}

ciudad de cultura científica: el $23,02 \%$ se declaran muy identificados y el 43,6\% identificados con ese objetivo, mientras que solo el $21,19 \%$ se declara poco identificado, el $5,34 \%$ dice estar nada identificado y a solo el $4,57 \%$ le resulta indiferente esa meta. [8]

Los entrevistados consideran que ese nivel de identificación se corresponde con las potencialidades identificadas en la ciudad y el hecho de que ese objetivo representa "una gran oportunidad para toda la provincia a nivel de imagen, desarrollo y proyección de futuro y económico". De igual forma, se consideran los posibles beneficios socioeconómicos que se asocian a tal propósito, así como también los vinculados a la dimensión personal y profesional en quienes tienen un vínculo laboral con la actividad científica.

En el caso de los argumentos ofrecidos desde una posición de baja o nula identificación con este propósito, apuntan tanto a la dimensión comunicativo-informativa como al compromiso y la implicación con el mismo, en ambos casos desde la perspectiva local. Así, se considera que existe falta de información o conocimientos para alcanzar ese objetivo o que no se observa una clara identificación de la población con una proyecto de este tipo, por lo cual se considera difícil acercar a la población turolense a este tema y motivarla/movilizarla en torno al mismo, generando un sentido de pertenencia e implicación al respecto. Las dimensiones comunicativa y emocional, ambas en relación con el escenario local, sintetizan este posicionamiento negativo respecto a hacer de Teruel una ciudad de cultura científica.

Sin embargo, la complejidad inherente a la cuestión local -dada su multidimensional y, en particular, su articulación de lo emotivo y lo racional- se confirma con el hecho de que, a pesar de esa presencia de personas que se declaran escasamente identificadas con esa meta, al mismo tiempo, hay una opinión abrumadoramente mayoritaria $(85,52 \%)$ de que Teruel debería apostar por el desarrollo científico y tecnológico como motor de desarrollo local. Se trata un porcentaje muy similar al de los españoles que, según la VI Encuesta de Percepción Social de la Ciencia (FECYT, 2012), piensan que la ciencia aporta ventajas para mejorar la calidad de vida de la sociedad $(88,6 \%)$ y opinan que contribuye al desarrollo económico $(87,1 \%)$.

Al mismo tiempo, la tensión entre individuo y colectivo, se muestra de manera particular en la mediación de lo local en los procesos comunicativos de configuración de la cultura científica. La diferencia de casi 20 puntos porcentuales entre quienes consideran que Teruel debería apostar por el desarrollo científico como motor de desarrollo local $(85,52 \%)$ con respecto a los que declaran sentirse identificados o muy identificados con hacer de Teruel una ciudad de cultura científica $(66,62 \%)$ confirma que, para ciertos sujetos, esa apuesta no se ve como una cuestión que los implique de manera directa e individual. Hay en este caso una similitud en ese comportamiento distinto entre lo social-institucional y lo personal, con la tendencia nacional a considerar de manera mayoritaria que las instituciones públicas deben dedicar más recursos a la ciencia, pero apenas el $40 \%$ de los españoles, de manera individual, estaría dispuesto a donar dinero a la ciencia (FECYT, 2012).

En tal sentido, son significativas las diferencias entre las respuestas a qué hacer de forma colectiva e individual para contribuir al objetivo de consolidar la actividad científica en Teruel. Desde el punto de vista personal, vuelve a considerarse un obstáculo el desconocimiento, tanto en lo relativo al tejido de las instituciones científicas locales, como de formación general.

Los entrevistados destacan además la influencia que ejerce la imagen de "expericidad" asociada a la ciencia, con lo cual quienes no tienen una vinculación laboral con esta área, sentirán que escapa a sus posibilidades personales hacer alguna aportación. Los sujetos percibirían una distancia personal 


\section{Revista Latina de Comunicación Social \# 069 - Páginas 618 a 636 \\ Investigación financiada | DOI: 10.4185/RLCS-2014-1027 | ISSN 1138-5820 | Año 2014}

respecto a la actividad científica y, en consecuencia, si bien se identifican con un proyecto local que incluya la misma, sienten que su participación o aportación al mismo es limitada.

Es relevante que los propios individuos reconozcan la necesidad de una mayor implicación personal, que se exprese en una mayor participación en las actividades de carácter científico que se realicen. Dos posibles contribuciones individuales señalada con frecuencia, serán precisamente aquellas que no requieren de nivel de experticidad o vinculación profesional con la actividad científica.

La primera será de naturaleza comunicativa -difundiendo, a través de las redes sociales propias (amigos, familiares), información sobre aquellas actividades locales vinculadas a la ciencia y la tecnología, así como de sus resultados-, y la segunda, de carácter emocional -coadyuvar en la sensibilización del resto de los turolenses con esta idea, animando a su entorno más inmediato y aportando en la construcción colectiva de una dimensión emocional, considerada imprescindible por los entrevistados para avanzar en este propósito.

Tanto el compromiso como la aportación individual y colectiva tienen, en consecuencia, un importante componente emotivo e identitario, dimensiones relevantes de lo local. Así, los entrevistados destacan la importancia de que "la ciudad se crea que tiene ese potencial" a la vez que "se crea en las posibilidades de la ciencia y la técnica".

De igual modo, se subraya la importancia de lograr una mayor cercanía de la población con la esta actividad, pues "en general tiene una idea bastante alejada de la realidad de lo que es la ciencia y los científicos", para lo cual se recomienda "poner en marcha actuaciones donde el ciudadano se sintiera más implicado, así como realizar más transferencia de resultados".

También se considera como oportunidad que, "en Teruel la gente es bastante participativa", lo cual facilitaría la implicación de sus habitantes. En fin, se señala que, en este proceso, "primero [hay que] lograr una conciencia clara de lo que significa esto. Empezar desde cero en la información al ciudadano y sobre todo, hacer sentir al turolense como algo suyo los avances que se puedan conseguir. (...) Simplemente informar a los ciudadanos de los pequeños aportes que logramos en el campo científico para que el ciudadano turolense se sienta orgulloso de su ciudad".

Las personas que se identifican con el propósito de hacer de Teruel una ciudad de cultura científica, tienen entre sus motivaciones fundamentales sus sentimientos hacia la localidad, sus deseos de que la misma crezca y sean conocidos los aspectos positivos de la misma -como los resultados de sus instituciones científicas-, o su compromiso con todo tipo de actuación que pretenda "hacer de mi querido Teruel algo mejor". En numerosos casos se señala la necesidad de hacer una mayor labor de divulgación hacia el exterior del entorno turolense que, como en el caso de la publicidad de esta actividad, mejoren la imagen de la ciudad.

Pero, como se ha señalado previamente, los sujetos insisten en la necesidad de que sea un proceso esencialmente colectivo, liderado desde las instituciones públicas, las empresas privadas y los centros científicos del territorio, reforzando la idea de una aportación individual entendida como colaboración con estas instituciones y las actividades realizadas por las mismas.

En ese deber-hacer colectivo se subraya de manera especial la dimensión económica, al demandarse una mayor financiación tanto pública como privada de la actividad de I+D+i. Esto confirma, en el caso analizado, la tendencia nacional a considerar necesario, de forma mayoritaria, que las administraciones públicas incrementen o mantengan la inversión en ciencia y tecnología (FECYT, 2012). 


\section{Revista Latina de Comunicación Social \# 069 - Páginas 618 a 636 \\ Investigación financiada | DOI: 10.4185/RLCS-2014-1027 | ISSN 1138-5820 | Año 2014}

\section{Discusión y conclusiones}

La potencia explicativa de la teoría de la mediación comunicativa de Jesús Martín-Barbero ha sido destacada por diversos autores (Gámez, 2007), en particular como parte del análisis de los procesos de construcción de sentido (Alonso, 2010), siendo que la mediación se define, precisamente, como "el lugar desde donde se otorga el sentido a la comunicación" (Martín-Barbero, 1986: 145). En consecuencia, resulta una propuesta pertinente para examinar la mediación del sentido de lo local en la articulación de la comunicación pública y la percepción social de la ciencia como parte de los procesos de configuración de la cultura científica.

En el caso analizado, el análisis de los resultados ha mostrado que, efectivamente, la dimensión local se presenta como una mediación fundamental en la comunicación pública y en la percepción social de la ciencia que tienen los sujetos.

Las valoraciones acerca de la actividad científica local -su impacto social, las instituciones del territorio que se asocian a esta actividad, las principales contribuciones que debe hacer al desarrollo local o los objetivos que deben alcanzarse en relación con esta actividad-, muestran que, frente a una actividad tradicionalmente presentada como territorio exclusivo de expertos y escenario del conocimiento científico -en oposición al conocimiento común-, la cultura científica de los individuos se soporta, en gran medida, en la información sobre aquellas instituciones de su entorno local -por consiguiente, físicamente próximas- vinculadas (o que se asumen como vinculadas) a esta actividad, así como la percepción que se tiene de estas.

La capacidad de impacto de las instituciones científicas locales tiene más relevancia que la importancia general social de los conocimientos que generan en la percepción pública de la ciencia y en los discursos de los sujetos en torno a ella.

En relación con ello, los sujetos muestran un elevado interés informativo por la actividad científica y prefieren, para satisfacerlo, espacios de comunicación que se caracterizan por su bidireccionalidad, tanto de naturaleza tecnológica -como son la Internet y las redes sociales-, como de carácter presencial, asociados precisamente a un entorno local que permite la comunicación cara a cara o al periodismo local. Con ello, se abre una posible área de actuación, susceptible de ser analizada en investigaciones posteriores, para la denominada prensa de proximidad, cuyo destacado papel como vertebrador de las comunidades locales en las que se inserta y a las cuales dota de un relato propio ha sido destacado (Pardo Baldeón, 2013).

Esta tendencia se inserta en el contexto de un cambio en la comunicación pública de la ciencia, confirmando que los sujetos ya no se limitan a una posición de receptores pasivos, según un modelo difusionista, sino que reclaman acciones de comunicación y también se convierten en comunicadores en este tema. La preferencia por Internet y las redes sociales, con su inherente interactividad y multimedialidad, confirman ese cambio.

La mayor cercanía geográfica y simbólica con las instituciones locales vinculadas a esa actividad, hace que la configuración de la percepción social de la ciencia y la cultura científica de los sujetos remitan, de manera directa, al quehacer de aquellas, pero también que exista una mayor demanda a los medios locales en relación con la comunicación pública de la ciencia, o a las entidades públicas y privadas de la localidad sobre los recursos disponibles para esta actividad.

Al mismo tiempo, la condición local media la contribución que los sujetos se plantean realizar a los 


\section{Revista Latina de Comunicación Social \# 069 - Páginas 618 a 636 \\ Investigación financiada | DOI: 10.4185/RLCS-2014-1027 | ISSN 1138-5820 | Año 2014}

procesos de configuración de la científica colectiva, muy ligada precisamente a dos dimensiones centrales en lo local: la comunicativa -a través de las redes sociales y comunicativas locales de los individuos-y la emocional - movilizadora de sentimientos e identidades.

Esta mediación del sentido de lo local, sin embargo, no está exenta de contradicciones. Por una parte, la proximidad física de las instituciones científicas locales, no logra romper con la representación social de la ciencia como una actividad de expertos, alejada de la vida cotidiana e inaccesible para la mayoría de las personas. Ello explica la falta de correspondencia entre un elevado interés informativo de los sujetos por estos temas y unos bajos niveles de asistencia a los centros de cultura científica.

Llo local también media las interrelaciones entre lo individual y lo colectivo en la percepción social de la ciencia como parte de la conformación de la cultura científica. Si bien los sujetos reconocen la importancia social y económica de la dimensión científica y tecnológica, demandan una mayor aportación de esta al desarrollo local futuro y muestran un elevado compromiso con el propósito de hacer de su localidad una ciudad de cultura científica, la aportación individual de los sujetos no se asume de manera extensiva.

Esa identificación con convertir su localidad en una ciudad con cultura científica no se considera una cuestión que los implique de manera directa, responsabilizándose de forma marcada a lo colectivo básicamente, instituciones de la administración pública y del ámbito privado- de esa labor.

De hecho, uno de los argumentos ofrecidos para explicar un posicionamiento pesimista con respecto a la viabilidad de convertir a Teruel en una ciudad de ciencia, es la falta de interés y la dificultad para lograr la implicación de los turolenses en ese propósito. Al mismo tiempo, se considera esencial que una de las líneas de actuación al respecto de esa meta sea motivacional, en estrecha relación con la comunicación. Es decir, se reconoce la centralidad de la dimensión emocional como parte de los procesos de confirmación de la cultura científica, sobre la base del sentido de pertenencia hacia, el compromiso con y incluso el amor al territorio.

La dimensión local también ofrece un soporte importante para un mayor componente crítico en relación con la actividad científica y tecnológica, una cuestión que, como se ha señalado, está entre las causas de la emergencia de un campo de estudio sobre percepción social de la ciencia y de comunicación pública de la misma. Si, como se ha planteado (Gil y Vilches, 2006), uno de los principales aportes de la ciencia a la cultura ciudadana es su contribución al desarrollo del espíritu crítico de los sujetos, entonces se puede considerar que la dimensión local, en este caso, actúa como reforzamiento de esa contribución, puesto que el sentido de pertenencia local acentúa la crítica -tanto en sentido negativo como propositivo- a la dimensión científica y tecnológica de la realidad cotidiana inmediata de su ciudad.

En tal sentido, la crítica aparece matizada precisamente por esa inmediatez y, en lugar de apuntar a cuestiones de ética u otros relatos universales, se centra en cuestiones como el financiamiento de esta actividad, su presencia en la opinión pública local, o la focalización de las instituciones locales hacia un público externo (visitantes), reclamando una mayor atención a los habitantes de la ciudad.

La responsabilización institucional, predominante frente a la responsabilidad individual, mostraría una cierta deformación en la lectura de lo local y lo colectivo asociado a ello, que se iguala a lo público y a la administración local. Ello dibuja un escenario en la cual esta lectura de lo local deviene una mediación central en la articulación entre participación y cultura científica.

El sentido de local contribuye tanto a la identificación de las sujetos con la actividad científica y con 


\section{Revista Latina de Comunicación Social \# 069 - Páginas 618 a 636}

Investigación financiada | DOI: 10.4185/RLCS-2014-1027 | ISSN 1138-5820 | Año 2014

el desarrollo local basado en ella, como a la responsabilización de las instituciones públicas y privadas locales con un mayor impacto de esa actividad. Si, efectivamente, la cultura científica no es un atributo individual sino social (Domínguez-Gutiérrez, 2006), entonces ello explica esa centralidad de la mediación de lo local en la configuración de aquella y de los procesos de comunicación pública y percepción social que se articulan en sus procesos de conformación, sobre todo en un contexto como el analizado, donde lo local tiene unas especiales importancia y densidad.

- Investigación realizada como parte del proyecto europeo Platform of Local Authorities and Communicators Engaged in Science (PLACES), que se enmarca en el $7^{\circ}$ Programa Marco de Investigación de la Unión Europea (FP7-SIS 244449). El artículo presenta una parte de los resultados de la consulta ciudadana realizada como parte del diseño del Plan de Acción Local del City Partnership de Teruel.

Fechas:

- inicio de la investigación: Enero de 2013

- término de la investigación: Mayo de 2013

\section{Notas}

[1] La cifra de 174.777 visitas recibidas en Dinópolis durante el año 2013 fue difundida por el gabinete de prensa de la Sociedad Gestora del Conjunto Paleontológico de Teruel y recogida, entre otros medios de comunicación, por Aragón Radio: "Dinópolis cierra la temporada con las mejores cifras de los últimos 9 años con cerca de 175.000 visitantes",12 de diciembre de 2013. Disponible en $<$ http://www.aragonradio2.com/noticias/hemeroteca/dinopolis-cierra-la-temporada-con-las-mejorescifras-de-los-ultimos-9-anos-con-cerca-de-175000-visitantes/> [Consultado el 1 de abril de 2014].

[2] El 0,15\% de los encuestados no respondió esta pregunta.

[3] El 1,07\% de los encuestados no responden a esta pregunta.

[4] No responden a esta pregunta el $0,46 \%$ de los encuestados.

[5] Movimiento social surgido en 1999, de la confluencia de varias plataformas (En defensa del Ferrocarril, Pro Salud Mental, Pro Helicóptero y Transporte Sanitario), en demanda de inversiones e infraestructuras que permitieran a Teruel alcanzar el nivel de desarrollo del resto de las provincias españolas. Este movimiento ciudadano logró aunar a la sociedad civil turolense en varios paros e incluso una huelga general que fue secundada ampliamente en toda la provincia.

[6] El 1,37\% de los encuestados no responde a esta pregunta.

[7] Estas cifras han sido publicadas por el Gobierno de Aragón, a partir de los datos del Servicio Público de Empleo Estatal (SEPE). Disponible en:

<https://www.aragon.es\%2Festaticos\%2FGobiernoAragon\%2FOrganismos\%2FInstitutoAragonesEst adistica\%2FDocumentos\%2Fdocs\%2FAreas\%2FEconomia\%2FInfCoy\%2Fparoreg.xls\&ei=SWZFU 7rlIq38yAH2woDIDg\&usg=AFQjCNFcfo5QdUznV4kr3ZOgFURGfPlyKw\&bvm=bv.64507335,d.a

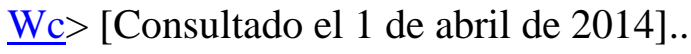

[8] El 2,29\% de los encuestados no responde a esta pregunta. 


\section{Referencias bibliográficas}

Aladro Vico, E., Padilla Castillo, G., Requeijo Rey, P., Semova, D.J., García Agustín, J, García Nieto, M.T., Viñarás Abad, M. (2014). "La presencia y representación de la mujer científica en la prensa española". Revista Latina de Comunicación Social, 69, pp. 176-194. http://www.revistalatinacs.org/069/paper/1007_UCM2/10g.html DOI: $10.4185 /$ RLCS-2014-1007.

Alcíbar, M. (2009). “Comunicación pública de la tecnociencia: más allá de la difusión del conocimiento". Zer, 14(27), pp. 165-188. www.ehu.es/zer/hemeroteca/pdfs/zer27-08-alcibar.pdf.

Alonso, M. (2010). "Mediación y Construcción de Sentidos: notas en torno a su articulación teóricometodológica en el estudio de la apropiación de Internet". Mediaciones Sociales. Revista de Ciencias Sociales y de la Comunicación, 6, pp. 3-37.

<http://revistas.ucm.es/index.php/MESO/article/view/MESO1010120003A>.

Alvira Martín, F. (2011). "La encuesta: una perspectiva general metodológica" Cuadernos Metodológicos 35. Madrid. CIS.

Cantalapiedra, M.J. (1997). "Periodistas locales”. Zer, 2(3), pp. 169-182. https://www.ehu.es\%2Fzer\%2Fhemeroteca\%2Fpdfs\%2Fzer03-11cantalapiedra.pdf\&ei=jBtGU5iXHqLCyQH37oFg\&usg=AFQjCNHcpYxN5Pf5Qe0RVRyW5A3eR $\underline{\text { WNkPA }}$

Creswell, J.W. y Plano Clark, V. (2011). Designing and Conducting Mixed Methods Research. Thousand Oaks, CA. Sage Publications.

Domínguez-Gutiérrez, S. (2006). "Las representaciones sociales en los procesos de comunicación de la ciencia", Actas I Congreso Iberoamericano de Ciencia, Tecnología, Sociedad e Innovación CTS + I. México: Palacio de Minería, 19-23 de junio de 2006.

<http://www.oei.es/memoriasctsi/mesa5/m05p21.pdf $>$.

Fayard, P. (2004). La comunicación pública de la ciencia. Hacia la sociedad del conocimiento. México. Dirección General de Divulgación de la Ciencia, UNAM.

FECYT (2012). VI Encuesta de Percepción Social de la Ciencia 2012. Madrid. FECYT.

Font, J., Blanco, I., Gomà, R. y Jarque, M. (2002). "Mecanismos de participación ciudadana en la toma de decisiones locales: una visión panorámica". $<$ http://www.opportugal.org/recursos.php?id=465>.

Gámez, N. (2007). "El paradigma de la mediación: crítica y perspectivas". Mediaciones Sociales. Revista de Ciencias Sociales y de la Comunicación, $\mathrm{n}^{\circ}$ 1, pp. 195-213. <http://revistas.ucm.es/index.php/MESO/article/view/MESO0707110195A〉.

García Galindo, J.A y Moreno Castro, C. (1999). "CTS y medios de comunicación social: algunas perspectivas para su análisis”. Zer, 6, pp. 219-231. <www.ehu.es/zer/hemeroteca/pdfs/zer06-11garcia.pdf>.

Gil, D. y Vilches, A. (2004). "La contribución de la ciencia a la cultura ciudadana”. Cultura y 
Educación, 16(3), pp. 259-272. DOI: 10.1174/1135640042360924

Gil, D. y Vilches, A. (2006). "Educación ciudadana y alfabetización científica: Mitos y realidades". Revista Iberoamericana de Educación, 42, pp. 31-53.

Gil, D., Macedo, B., Martínez Torregrosa, J., Barrios, C., Valdés, P. y Vilches, A. (2005). ¿Cómo promover el interés por la cultura científica? Una propuesta didáctica fundamentada para la educación científica de jóvenes de 15 a 18 años. Santiago de Chile. Oficina Regional de Educación de la UNESCO para América Latina y el Caribe.

Hylland Erikse, T. (2014). Globalization: The Key Concepts. London/New York. Bloomsbury Publishing Plc.

Instituto Nacional de Estadísticas INE (2009). Cifras INE. Boletín Informativo del INE, 5/2009. 11 de julio de 2009. 〈http://www.ine.es/>.

Instituto Nacional de Estadísticas INE (2013). Estadística del Padrón Continuo a 1 de enero de 2013. Datos por municipios. <http://www.ine.es/>.

López Cerezo, J. A., Cabello Valdés, C. Muñoz Vivas, L. y Polino, C. (2009). Cultura científica en Iberoamérica. Encuesta en grandes núcleos urbanos. Proyecto Estándar Iberoamericano de Indicadores de Percepción Pública, Cultura Científica y Participación Ciudadana (2005-2009). Madrid. FECYT, OEI, RICYT.

Magro Mazo, C. (2008). "Plan Nacional y cultura científica. El Plan Nacional de I+D+i (2008-2011) a examen", mi+d Revista, 21, pp.141-154.

Martín-Barbero, J.(1986). De los medios a las mediaciones. México. Gily.

Martínez Ruiz, F.J., Bautista Arnedo, M.M, Del Pino Ruiz, J.R. (2005). "Educación científica, sociedad y televisión". Revista Comunicar, Vol. XIII, 25.

$\langle$ http://www.revistacomunicar.com/verpdf.php?numero=25\&articulo=25-2005-203 $>$.

Morley, D. y Robins, K. (2013). Spaces of Identity: Global Media, Electronic Landscapes and Cultural Boundaries. London/New York. Routledge.

Pardo Baldeón, R.(2013). "Las aportaciones de la prensa de proximidad a la formación de comunidades locales. Veintidós años de Levante de Castelló". Historia y Comunicación Social, 18. $\langle$ http://revistas.ucm.es/index.php/HICS/article/view/44315/41873>.

Pellegrino, G. (2011). The Politics of Proximity: Mobility and Immobility in Practice, Farnham, UK. Ashgate Publishing.

Piñuel Raigada, J.L (2002). "Epistemología, metodología y técnicas del análisis de contenido". Estudios de Sociolingüística, 3 (1), España, Universidad Complutense de Madrid, pp. 1-42.

Polino, C.,_Fazio, M.E. y Vaccarezza, L. (2003). "Medir la percepción pública de la ciencia en los países iberoamericanos. Aproximación a problemas conceptuales". Revista Iberoamericana de Ciencia, Tecnología, Sociedad e Innovación, 5.

$<$ http://www.oei.es/revistactsi/numero5/articulo1.htm>.

Salcedo, M. (2009). "El antropomorfismo como herramienta de divulgación científica por televisión: estudio de El Hombre y la Tierra". Comunicación y Sociedad, Vol. XXIV, 1, pp. 217-246. 
Vaccarezza, L. S. (2008). "Exploraciones en torno al concepto de cultura científica", Resúmenes del Congreso Iberoamericano de Ciudadanía y Políticas Públicas de Ciencia y Tecnología. Madrid. FECYT.

\section{Artículos relacionados}

Cazaux, D. (2008). "La comunicación pública de la ciencia y la tecnología en la sociedad del conocimiento". Razón y Palabra, 13(65), noviembre-diciembre, Instituto Tecnológico y de Estudios Superiores de Monterrey, México. 〈http://www.redalyc.org/articulo.oa?id=199520724004〉.

Bensaude-Vincent, B. (2001). "A genealogy of the increasing gap between science and the public". Public Understanding of Science, 10(1), pp. 99-113. DOI: 10.1088/0963-6625/10/1/307

\section{Cómo citar este artículo / Referencia normalizada}

A Sanz-Hernández, L Alcalá-Martínez, L Bacallao-Pino (2014): “Comunicación pública de la ciencia, cultura científica y sentido de localidad. El caso de la ciudad de Teruel, España". Revista Latina de Comunicación Social, 69, pp. 618 a 636.

http://www.revistalatinacs.org/069/paper/1027_UNAM/30es.html

DOI: $\underline{10.4185 / R L C S-2014-1027}$

Artículo recibido el 28 de agosto de 2014. Aceptado el 2 de octubre. Publicado el 8 de octubre. 